\title{
Electron dispersive X-ray spectroscopy and degradation properties of hyaluronic acid decorated microparticles
}

\section{Serri, Carla}

2019-09-01

Serri , C , Frigione , M , Ruponen, M , Urtti , A , Borzacchiello , A , Biondi , M , Itkonen , J \& Mayol , L 2019 , ' Electron dispersive X-ray spectroscopy and degradation properties of hyaluronic acid decorated microparticles ' , Colloids and Surfaces B: Biointerfaces , vol. 181 , pp. 896-901 . https://doi.org/10.1016/j.colsurfb.2019.06.044

http://hdl.handle.net/10138/331640

https://doi.org/10.1016/j.colsurfb.2019.06.044

cc_by_nc_nd

acceptedVersion

Downloaded from Helda, University of Helsinki institutional repository.

This is an electronic reprint of the original article.

This reprint may differ from the original in pagination and typographic detail.

Please cite the original version. 


\section{Accepted Manuscript}

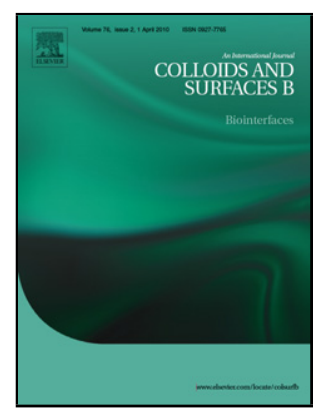

Title: Electron dispersive X-ray spectroscopy and degradation properties of hyaluronic acid decorated microparticles

Authors: Carla Serri, Mariaenrica Frigione, Marika Ruponen, Arto Urtti, Assunta Borzacchiello, Marco Biondi, Laura Mayol

PII: S0927-7765(19)30450-3

DOI: https://doi.org/10.1016/j.colsurfb.2019.06.044

Reference: COLSUB 10314

To appear in: Colloids and Surfaces B: Biointerfaces

Received date: $\quad 5$ March 2019

Revised date: $\quad 28$ May 2019

Accepted date: 18 June 2019

Please cite this article as: Serri C, Frigione M, Ruponen M, Urtti A, Borzacchiello A, Biondi M, Mayol L, Electron dispersive X-ray spectroscopy and degradation properties of hyaluronic acid decorated microparticles, Colloids and Surfaces B: Biointerfaces (2019), https://doi.org/10.1016/j.colsurfb.2019.06.044

This is a PDF file of an unedited manuscript that has been accepted for publication. As a service to our customers we are providing this early version of the manuscript. The manuscript will undergo copyediting, typesetting, and review of the resulting proof before it is published in its final form. Please note that during the production process errors may be discovered which could affect the content, and all legal disclaimers that apply to the journal pertain. 


\title{
Electron dispersive $\mathrm{X}$-ray spectroscopy and degradation
}

\section{properties of hyaluronic acid decorated microparticles}

\author{
Carla Serri ${ }^{1}$, Mariaenrica Frigione ${ }^{2}$, Marika Ruponen ${ }^{3}$, Arto Urtti ${ }^{3,4}$, Assunta \\ Borzacchiello $^{5}$, Marco Biondi ${ }^{1,6, *}$, Laura Mayol ${ }^{1,6}$
}

${ }^{1}$ Dipartimento di Farmacia, Università di Napoli Federico II, Via D. Montesano 49, Napoli, Italy.

${ }^{2}$ Dipartimento di Ingegneria dell'Innovazione - Università del Salento - S.P. 6, Lecce - Monteroni

- Lecce, Italy

${ }^{3}$ School of Pharmacy, Faculty of Health Sciences, University of Eastern Finland, 70211 Kuopio,

Finland

${ }^{4}$ Centre for Drug Research, Division of Pharmaceutical Biosciences, Faculty of Pharmacy, University of Helsinki, P.O. Box 56, FI-00014 Helsinki, Finland

${ }^{5}$ Istituto per i Materiali Compositi e Biomedici (IMCB-CNR), Università di Napoli Federico II, Viale J.F. Kennedy, Napoli, Italy

${ }^{6}$ Interdisciplinary Research Centre on Biomaterials - CRIB - Università di Napoli Federico II - P.le Tecchio, 80, Napoli, Italy

${ }^{*}$ Correspondence to:

Marco Biondi, Dipartimento di Farmacia, Scuola di Medicina e Chirurgia, Università di Napoli Federico II, Via D. Montesano 49, Napoli, Italy.

mabiondi@unina.it

Graphical abstract 


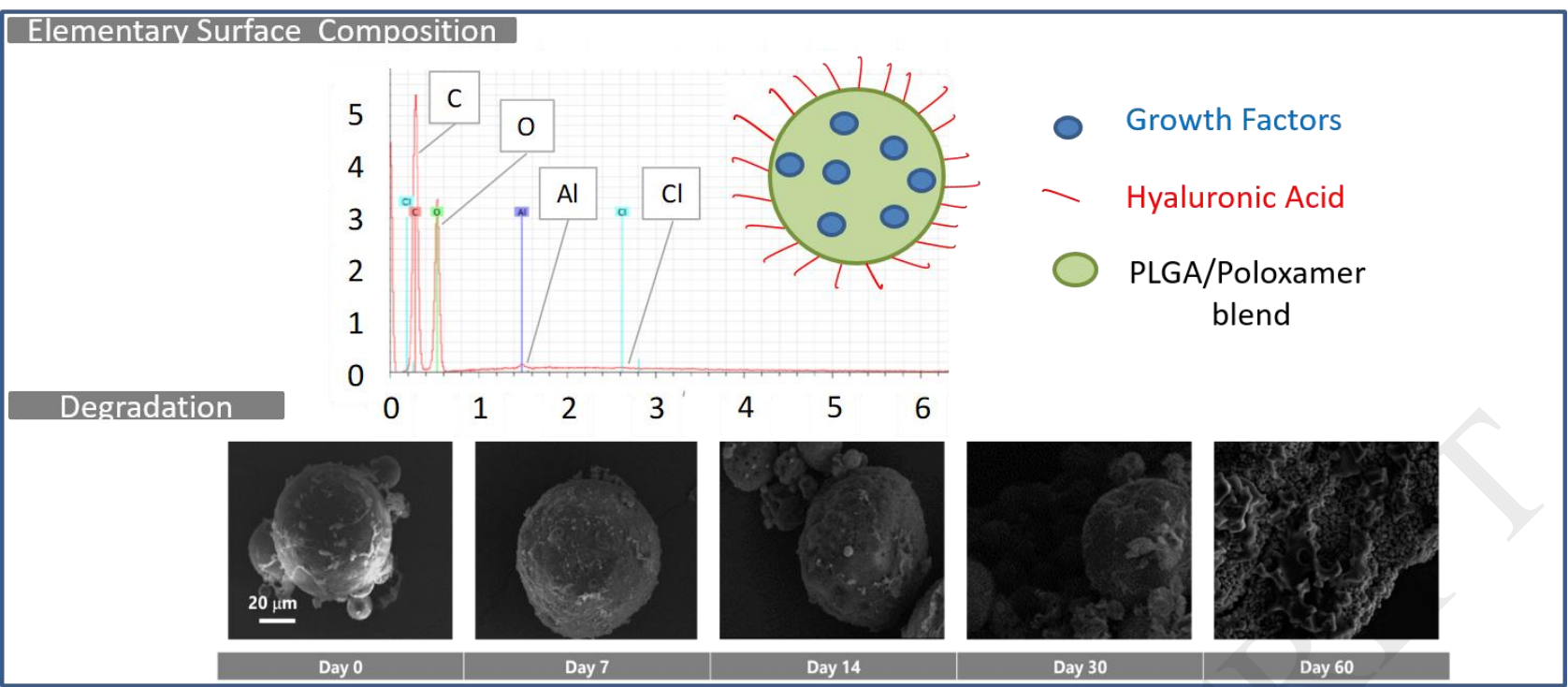

\section{Highlights}

- Microparticles based on PLGA/poloxamers have been decorated with hyaluronic acid with no chemical reaction

- EDS results indicate the migration of ethylene oxide segments of poloxamers towards microparticle surface

- Poloxamers only partially blend in PLGA-containing bulk of microspheres

- Microparticle degradation is strongly slowed down in the presence of poloxamers

\section{Abstract}

The purpose of this study was to produce poly(DL-lactic-co-glycolic acid) (PLGA) - based microparticles (MPs), externally decorated with hyaluronic acid (HA). The MPs are intended for intravitreal injections in the treatment of posterior eye segment and have been designed to prolong the release of growth factors into the vitreous body, therefore aiming to increase the time interval between two consecutive injections. The MPs, prepared by a modified double emulsion-solvent evaporation technique and loaded with bovine serum albumins (BSA) and ciliary neurotrophic factor (CNTF), were spherical, with a diameter around $70 \mu \mathrm{m}$ and $\mathrm{a}>90 \%$ encapsulation efficiency. Energy Dispersive Spectroscopy (EDS) outcomes indicated that HA presence in the external aqueous phase of the emulsion did affect the surface properties of MPs. Moreover, poloxamers drastically slowed 
down MP degradation properties which are, in turn, closely related to their ability to prolong drug release. This is promising for the envisaged application of the produced MPs. Further work will be devoted to optimizing MP formulation with respect to the envisaged intravitreal route of administration.

Keywords: hyaluronic acid, microparticle, PLGA, poloxamer, diffusion, ocular delivery.

\section{Introduction}

Diseases of the posterior eye segment are often related to elderly and are elicited by a pathological angiogenesis which can lead to blindness [1,2]. Furthermore, due to continuous global aging, a significant increase in the prevalence of these diseases is expected in the near future [3]. Currently, the only route of administration that can most effectively ensure therapeutic drug concentrations in the posterior part of the eye is the intravitreal injection [4]. For example, the wet form of age-related macular degeneration is effectively treated with injections of antiangiogenic antibodies directly in the vitreous body, generally every 1-3 months [5-7]. Unfortunately, this route of administration is very invasive and is associated to a high risk of endophthalmitis, cataracts, retinal detachment and vitreous hemorrhage [8], and also causes significant costs for the health care system $[9,10]$. In this sense, there is a pressing need to extend the time between two consecutive injections to delay or discourage the onset of adverse effects. The production of injectable systems able to sustain drug release is expected to respond to this urgent and unmet clinical need. In this context, one attractive approach relies upon the use of biodegradable microparticles (MPs) based on poly(lactic-co-glycolic acid) (PLGA), which is a first-choice polyester to this aim due to its approval by FDA for human use [11-14].

It must be underlined that, for an effective intravitreal treatment, MPs should not aggregate after injection. Indeed, this results into a reduction of the exposed area of the devices and, consequently, to a less effective control over the kinetics of drug delivery. In this perspective, the superficial decoration of MPs with hyaluronic acid (HA) is particularly interesting. Indeed, HA is one of the 
main constituents of human vitreous humor [15], and its presence on MP surface increase the affinity between the MPs and the vitreous body.

Recently, we have produced PLGA-based nanoparticles coated with hydrophilic HA for the active targeting of chemotherapeutic drugs by emulsion [16] or nanoprecipitation technique [17]. In these two works, HA coating was physically obtained using amphiphilic poloxamers as 'bridging' molecules between hydrophobic PLGA and hydrophilic HA. In the present work, we aimed to translate the same production technique from the nano- to the micro-scale. For this purpose, HAcoated MPs based on a PLGA/poloxamers organic blend were produced by a slightly modified double emulsion-solvent evaporation technique. The obtained MPs were loaded with BSA and Ciliary neurotrophic factor (CNTF) [18] and characterized for their technological and thermodynamic features, and their surface properties investigated by Energy Dispersive Spectroscopy (EDS). Finally, the composition of MPs was correlated to their degradation properties which are, in turn, closely related to their ability to prolong drug release.

\section{Materials and Methods}

\subsection{Materials}

HA with a weight-average molecular weight (MW) of 734,000 Da was provided by Lifecore Biomedical (USA). Equimolar uncapped poly (D,L-lactic-co-glycolic acid) (PLGA) (Resomer RG504H, Mw $40 \mathrm{kDa}$, inherent viscosity: 0.16-0.24 $\mathrm{dL} \mathrm{g}^{-1}$ in acetone at $25^{\circ} \mathrm{C}$ ) from Evonik (Germany) was used. Poloxamers $\left(\mathrm{PEO}_{\mathrm{a}}-\mathrm{PPO}_{\mathrm{b}}-\mathrm{PEO}_{\mathrm{a}}\right)$, which are amphiphilic triblock polymers containing different numbers of oxyethylene (a) and oxypropylene (b) units, were used. In this work, poloxamers F127 $(\mathrm{a}=100$ and $\mathrm{b}=65)$ and F68 $(\mathrm{a}=76$ and $\mathrm{b}=29)$ from LUTROL (Basf, Germany) were employed. Ciliary neurotrophic factor (CNTF) synthesized according to a previously reported procedure was used [19]. All other chemicals, i.e. Bovine serum albumin (BSA), Ethanol (EtOH), acetone, dimethylsulfoxide (DMSO), dichloromethane (DCM), poly (vinyl alcohol) (PVA: Mowiol 
40-88), phosphate buffer salts, sodium e potassium chloride (PBS) were obtained from SigmaAldrich (USA).

\subsection{Microparticle production}

MPs loaded with BSA and CNTF were prepared with a theoretical protein loading of $0.20 \%(0.20$ mg of BSA only per $100 \mathrm{mg}$ of microparticles), using double emulsion-solvent evaporation technique [20-22], with slight modifications applied herein. Briefly, $0.25 \mathrm{~mL}$ of an internal aqueous phase consisting of a BSA/CNTF solution $(0.4 \% \mathrm{w} / \mathrm{v}$ overall protein content with respect to the internal aqueous phase; BSA:CNTF mass ratio was set to 20:1) in phosphate buffer (PBS, $1.420 \mathrm{~g}$ of $\mathrm{Na}_{2} \mathrm{HPO}_{4}, 0.201 \mathrm{~g}$ of $\mathrm{KCl}$ and $7 \mathrm{~g}$ of $\mathrm{NaCl}$ in $1 \mathrm{~L}$ of purified $\mathrm{H}_{2} \mathrm{O}$ ) were poured into an organic solution made of $250 \mathrm{mg}$ of PLGA, $125 \mathrm{mg}$ of poloxamer F68 and $125 \mathrm{mg}$ of poloxamer F127 in 2.5 $\mathrm{mL}$ of dichloromethane (DCM). The two phases were then emulsified by a high-speed homogenizer (Diax 900, equipped with a 10G probe, Heidolph, Germany; 11,000 rpm, $2 \mathrm{~min}$ ) and the obtained water-in-oil primary emulsion was poured in $40 \mathrm{~mL}$ of an external aqueous solution further homogenized at 11,000 rpm (10G probe) for 2 minutes, to obtain the final double emulsion. The second aqueous phase contained 7.5 mg of F68 and 7.5 mg of F127 and, only for HA-decorated MPs (namely PPHA), $30 \mathrm{mg}$ of hyaluronic acid. The organic solvent was then evaporated overnight under magnetic stirring (MR $3001 \mathrm{~K}$, Heidolph, Germany) at room temperature for MP hardening, which were finally centrifuged $\left(5000 \mathrm{rpm}, 4^{\circ} \mathrm{C}\right)$, washed with distilled water three times (Universal 16R, Hettich Zentrifugen, Germany) and lyophilized for 24 hours $\left(0.01 \mathrm{~atm},-60^{\circ} \mathrm{C}\right)$ (Modulyo, Edwards, United Kingdom). Control MPs (namely PP) were also produced in the absence of HA in the external water phase.

\subsection{Microparticle size}

The mean volume and size distribution of the produced MPs were determined by light scattering (Microtrac S3500 Series Particle Size Analyzer; Montgomeryville, PA, USA). The tests were run by 
dispersing lyophilized MPs in MilliQ Water containing 0.5\% w/v PVA. The results and the standard deviation have been averaged on triplicate samples.

\subsection{Yield and entrapment efficiency of microparticles}

The loaded BSA/CNTF were quantified by the direct method, by dissolving the MPs in $\mathrm{NaOH} 0.1 \mathrm{~N}$ $(10 \mathrm{mg} / \mathrm{mL})$ under continuous stirring for about $5 \mathrm{~h}$. Subsequently, the obtained solution was analyzed for protein content by High-Performance Liquid Chromatography (HPLC). The chromatograph was equipped with a HPLC LC-20A pump (Shimadzu), a 7725i injection valve (Rheodyne), a SPV-10A UV-vis detector (Shimadzu) set at the wavelength of 214/220 $\mathrm{nm}$ and a C-R6A integrator (Shimadzu). Chromatographic separation was realized by a gradient elution applied at a flow rate of $1 \mathrm{~mL} / \mathrm{min}$ in a Phenomenex Luna C4 $300 \AA$ A column (4.6 mm inner diameter, $5.0 \mu \mathrm{m}$ particles; injection volume: $20 \mu \mathrm{L} ; \lambda=220 \mathrm{~nm}$ for BSA and $\lambda=214 \mathrm{~nm}$ for CNTF). Trifluroacetic acid (TFA) was added to a mixture of water and acetonitrile, at a $0.1 \%$ volume fraction in the mobile phase. Gradient elution was optimized for solution A (900 mL of water, $100 \mathrm{~mL}$ acetonitrile, $1 \mathrm{~mL}$ TFA) and solution $\mathrm{B}(900 \mathrm{~mL}$ of acetonitrile, $100 \mathrm{~mL}$ water, $1 \mathrm{~mL}$ TFA), and it was found out that 5 to $50 \%$ of solution B in 15 minutes at a flow rate of $1 \mathrm{~mL} / \mathrm{min}$ allowed an optimized separation of BSA and CNTF. The following gradient was applied: B 10\% (0-2 min), B 50\% (2-5 min) B 90\% (5-12.5 min), B 10\% (12.5-15 min). Thus, actual loading was expressed as mg of encapsulated BSA or CNTF per $100 \mathrm{mg}$ of MPs, while the encapsulation efficiency was expressed as the ratio of actual and theoretical drug loading $\times 100 \pm$ standard deviation of values collected from at least three different batches.

The yield of MPs, expressed as percentage of the obtained mass of microdevices with respect to the theoretical expected mass, was gravimetrically determined from MP mass recovered after freezedrying (0.01 atm, 24 hours; Modulyo, Edwards, UK).

\subsection{Energy-dispersive $X$-ray spectroscopy (EDS)}


Elemental distribution of the constituent materials and MPs was investigated by using Zeiss Evo40/Energy-dispersive X-ray spectroscopy (EDS) at three different voltages (10, 15 and $20 \mathrm{kV})$. For these analyses, the powders were employed without any further modifications. Results are expressed as mean value \pm the standard deviation of at least two repeats.

\subsection{Degradation kinetics of MPs}

As described in previous publications [20,21], the degradation of the MPs was qualitatively studied in terms of the morphological variations occurring during degradation after suspending the MPs in a PBS solution and incubating the suspensions at $37^{\circ} \mathrm{C}$ on a gently oscillating base (15 rpm) (Stoval Life Science Inc., USA). At scheduled time intervals, the MPs were centrifuged, washed three times and lyophilized. Tiny aliquots of the freeze-dried, partially degraded samples were prepared by goldsputtering under vacuum and analyzed by Scanning electron microscopy (SEM; Jeol JSM-6335F, Tokyo, Japan) to observe the changes in time of the external shape and morphology of the degrading MPs.

\subsection{Differential scanning calorimetry}

The interactions among the polymers in PPHA MPs were studied by thermoanalytical tests, which were carried out on as-received raw materials (PLGA, HA, single poloxamers and physical mixture thereof) and on the freeze-dryed MPs. The thermal events related to the phase transitions of the samples were recorded by a differential scanning calorimeter (DSC, DSC Q20, TA Instruments, USA), previously calibrated with an indium standard. The samples were placed in aluminum capsules and heated from $-60^{\circ} \mathrm{C}$ to $80^{\circ} \mathrm{C}$ at $5^{\circ} \mathrm{C} / \mathrm{min}$ under an inert nitrogen atmosphere (flow rate: $50 \mathrm{~mL} / \mathrm{min}$ ). Double scans were performed, with an equilibration segment between the first and the second scan. All experiments were run in triplicate.

\section{Results and discussion}


In this study, PLGA/poloxamers MPs, externally decorated or not with HA, (named, respectively, PP and PPHA MPs) were fabricated with a modified double emulsion/solvent evaporation technique. Even if emulsion-based processing of MPs may induce protein inactivation and aggregation at w/o interface, great progresses have been made in formulation optimization and, to date, straightforward strategies for protein stabilization have been developed [23,24]. Among them, a widely employed approach involves the co-encapsulation of a surface-active protein, such as BSA, which displaces the therapeutic protein from w/o interface (i.e. sacrificial lamb approach). For this reason, we decided to co-encapsulate BSA with CNTF. Very high encapsulation efficiencies (EE) was obtained for both proteins: specifically, EE was $94.1 \pm 0.9 \%$ for BSA and $99.7 \pm 0.7 \%$ for CNTF. These results are in line with the outcomes of our previous publications dealing with protein loading in PLGA microparticles [20-22]. Formulation conditions of MPs were fixed so as to produce devices with a diameter significantly higher than the mesh size of the vitreous humor, which is known to be $<1 \mu \mathrm{m}$ in bovine [25]. This is important to discourage the transport of MPs within the vitreous, since free MP diffusion would hinder a correct vision. The diameter of the produced MPs was $(67.4 \pm 2.6)$ and $(73.4 \pm 5.3) \mu \mathrm{m}$, with a $(42.5 \pm 5.0)$ and $(36.5 \pm 0.8) \%$ yield percentages for PP and PPHA MPs, respectively. This indicates that, due to the large size of MPs, their diffusion is completely hindered in vitreous.

To investigate the superficial composition of MPs, EDS analyses were performed on the raw materials (HA, poloxamers and PLGA), as well as on PP and PPHA MPs. In both cases, the external surface of MPs is mainly composed by carbon and oxygen (Fig. 1 and Table 1). In particular, for PPHA MPs, carbon is slightly prevailing over oxygen. Traces of aluminum and chlorine were also detected; the latter were ascribed to possible residues of DCM after MP hardening. Results are highly reproducible and, as expected, the magnifications (from $300 \mathrm{X}$ to $3000 \mathrm{X}$ ) and voltages (10, 15 and $20 \mathrm{kV}$ ) employed for EDS tests had a negligible influence on the outcomes. The elemental composition of PLGA powder closely resembles the one of PP MPs. However, a slightly higher carbon percentage was 
found in this latter case, indicating that some poloxamers are present on MP surface, even if PLGA prevails at the micro-scale. In two previous works $[16,26]$, we found that, at the nanoscale, the presence of poloxamers did actually enhance the superficial hydrophilicity of nanoparticles based on PLGA and poloxamers and produced with the same technique. This indicates that the superficial arrangement of hydrophilic segments of poloxamers is enhanced at the nano-scale. Similarly, we have previously produced nanoparticles by dissolving HA in the dispersing phase used to produce the nanodevices, and we found out that HA was successfully deposited on nanoparticle surface. This was ascribed to the lipophilicity gradient established between the continuous (aqueous) and the dispersed (organic) phases used to produce the nanoparticles [16,17]. In this work, at micro-scale, PPHA MPs were produced similarly, by solubilizing hydrophilic HA in the external aqueous phase of the emulsion used for MP production. Interestingly, EDS results showed a slight increase in carbon percentage in this case, which hints at a different superficial arrangement of the polymers. This is indicative of a higher percentage of hydrophilic poloxamer segments at the surface of MPs and can be reasonably ascribed to an increased surface hydrophilicity of PPHA MPs, which in turn is driven by the interaction with HA molecules during the preparation of MPs.

DSC thermograms (1st and 2nd heating cycles) of poloxamers, physical mixtures thereof, PLGA, HA and PPHA MPs are shown in Figs. 2A and 2B, respectively. The thermograms for the first heating of poloxamers exhibited endothermic melting peaks, indicating their crystalline or semi-crystalline nature. In the case of poloxamers physical mixtures, two melting peaks were visible (Fig. 2A), although with a significant superimposition. The second scans displayed a low decrease of the melting points of poloxamers, along with the involved heats. In all cases, HA did not show any thermodynamic event in the investigated temperature range. In contrast, PLGA was amorphous and showed a glass transition temperature $(\mathrm{Tg})$ at about $52^{\circ} \mathrm{C}$ on the first heating run, also showing a typical endothermic relaxation peak which, as expected, was not observed in the second heating scan. In the case of microparticles, the melting peaks of poloxamers were less evident, indicating that these 
polymers, despite their ability to enhance HA superficial disposition, only partially blend within microparticle bulk. More specifically, as calculated by comparing the melting heat of microparticles and poloxamer mixtures, normalized with respect to the mass fraction of poloxamers, approximately $74 \%$ of the poloxamers are amorphized during microparticle preparation. The Tg of PLGA in MPs could not be detected in both heating cycles. Probably, the glass transition event has been masked by the melting peak of poloxamers, which were detected at a lower temperature compared to poloxamers alone. This indicates that, in the polymeric blend formed during MP formulation, PLGA and poloxamer were not mixed at the molecular level in the microparticles and formed a co-amorphous, partially phase-separated system. Moreover, the lower melting temperature indicates that the formation of large poloxamers crystals is discouraged and, more in detail, that part of the crystalline regions of the poloxamers are dissolved in the rubbery PLGA. In the second heating run, the evolved melting heats were drastically lowered, thereby showing that the re-organization of molten poloxamers in the rubbery/molten matrix of MPs is strongly discouraged in this case.

As described in Section 2.6, the morphological modifications over time of MPs during degradation have been studied in vitro over a two-month course by SEM observations. At $\mathrm{t}=0$, i.e. immediately after production, it can be clearly observed that MPs showed a well-rounded, spherical morphology and a non-porous shell, with small particles adhering to the surface (Fig. 3). After 14 days of incubation, minor changes occurred at the surface of MPs; after 30 days, significant changes in the original shape of MPs was observed and, only after 60 days, the structural integrity of MPs completely vanished. This has been compared to previous results, in which MP disruption was found to occur within shorter times (2-4 weeks) [20,27]. Actually, autocatalytic PLGA degradation can be schematized as follows: (i) upon contact with an aqueous medium, water intrudes the polymeric matrix of MPs through their meso- microporous structure [28], causing PLGA plasticization [29]; (ii) thereafter, hydrolysis-triggered PLGA degradation at chain backbone occurs, causing the formation of acidic by-products. They can diffuse slowly through the polymeric core of MPs, which encourages 
their accumulation within the internal regions of MPs and a consequent, substantial drop of local $\mathrm{pH}$ values [30,31]; (iii) the cleavage of the ester bonds is catalyzed by protons, thereby autoaccelerating PLGA degradation [32]. PLGA molecular weight steadily decreases, while device mass loss starts when polymer molecules reach a critical molecular weight below which PLGA is soluble.

In this work, PLGA has been blended with poloxamers in MP core, and this is the mainly responsible for the strong slowing of degradation kinetics. Indeed, the increased hydrophilicity of MPs due to poloxamers presence is expected to facilitate water penetration and PLGA degradation onset. On the other hand, this also enhances the transport of acidic degradation products out of the devices, thereby favouring their neutralization in the incubation medium, therefore discouraging autocatalysis. In the produced MPs, the results clearly indicate that the facilitated neutralization of acidic by-products overshadows the effect of accelerated water intrusion and elicits a slower polymer degradation, as recently demonstrated [32]. This indicated that the presence of poloxamers in the organic phase is mainly responsible for the slowing down of hydrolytic PLGA degradation compared to MPs made up of PLGA alone [19]. A delayed degradation of MPs, in turn, is associated to a sustained release of drug molecule(s), thereby reducing the frequency of intravitreal injections and the related risk of adverse effects. This is advantageous because it should reduce the release of potentially harmful acidic degradation products in the vitreous body and also to slow down the release kinetics of growth factors from MPs.

\section{Conclusions}

In the present study, MPs based on PLGA/poloxamers have been decorated with HA by using a slightly modified double emulsion/solvent evaporation technique. This technique has been chosen to translate to the micro-scale a previously optimized nanoprecipitation technique used to obtain amphiphilic nanoparticles based on a PLGA/poloxamers blend. The presence of HA and poloxamers increased MP hydrophilicity, facilitated by an enhanced migration of EO segments toward the 
external regions of the MPs, as indicated by EDS results. This was also corroborated by DSC results, which showed that poloxamers only partially blend in the organic phase of the emulsion, while their amorphized fraction probably exerts the bridging function between PLGA and HA. Furthermore, SEM images revealed that poloxamers strongly slow down the degradation of MPs through a complex balance between facilitated water intrusion, which triggers degradation, and enhanced transport of acidic by-products, which catalyze the degradation. Overall, this study implies the potential of chemical reaction-free HA decoration through poloxamers bridging onto PLGA MPs in promoting the overall surface hydrophilicity, which is in turn promising for the envisaged application for intravitreal injections.

Reference List

[1] M.N. Yasin, D. Svirskis, A. Seyfoddin, and I.D. Rupenthal, Implants for drug delivery to the posterior segment of the eye: a focus on stimuli-responsive and tunable release systems, J. Control Release, 196 (2014) 208-221.

[2] J. Araujo, S. Nikolic, M.A. Egea, E.B. Souto, and M.L. Garcia, Nanostructured lipid carriers for triamcinolone acetonide delivery to the posterior segment of the eye, Colloids Surf. B Biointerfaces., 88 (2011) 150-157.

[3] R. Klein and B.E. Klein, The prevalence of age-related eye diseases and visual impairment in aging: current estimates, Invest Ophthalmol. Vis. Sci., 54 (2013) ORSF5-ORSF13.

[4] P. Bansal, S. Garg, Y. Sharma, and P. Venkatesh, Posterior Segment Drug Delivery Devices: Current and Novel Therapies in Development, J. Ocul. Pharmacol. Ther., 32 (2016) 135-144.

[5] T.R. Thrimawithana, S. Young, C.R. Bunt, C. Green, and R.G. Alany, Drug delivery to the posterior segment of the eye, Drug Discov. Today, 16 (2011) 270-277.

[6] D.H. Geroski and H.F. Edelhauser, Drug delivery for posterior segment eye disease, Invest Ophthalmol. Vis. Sci., 41 (2000) 961-964.

[7] A. Patel, K. Cholkar, V. Agrahari, and A.K. Mitra, Ocular drug delivery systems: An overview, World J. Pharmacol., 2 (2013) 47-64.

[8] F.G. Holz, R. Tadayoni, S. Beatty, A. Berger, M.G. Cereda, R. Cortez, C.B. Hoyng, P. Hykin, G. Staurenghi, S. Heldner, T. Bogumil, T. Heah, and S. Sivaprasad, Multi-country real-life experience of anti-vascular endothelial growth factor therapy for wet age-related macular degeneration, $\mathrm{Br}$. J. Ophthalmol., 99 (2015) 220-226.

[9] R.D. Schoenwald, Ocular drug delivery. Pharmacokinetic considerations, Clin. Pharmacokinet., 18 (1990) 255-269. 
[10] A. Urtti, Challenges and obstacles of ocular pharmacokinetics and drug delivery, Adv. Drug Deliv. Rev., 58 (2006) 1131-1135.

[11] J.M. Lu, X. Wang, C. Marin-Muller, H. Wang, P.H. Lin, Q. Yao, and C. Chen, Current advances in research and clinical applications of PLGA-based nanotechnology, Expert. Rev. Mol. Diagn., 9 (2009) 325-341.

[12] F. Danhier, E. Ansorena, J.M. Silva, R. Coco, B.A. Le, and V. Preat, PLGA-based nanoparticles: an overview of biomedical applications, J. Control Release, 161 (2012) 505-522.

[13] N.B. Shelke, R. Kadam, P. Tyagi, V.R. Rao, and U.B. Kompella, Intravitreal Poly(L-lactide) Microparticles Sustain Retinal and Choroidal Delivery of TG-0054, a Hydrophilic Drug Intended for Neovascular Diseases, Drug Deliv. Transl. Res., 1 (2011) 76-90.

[14] S.K. Yandrapu, A.K. Upadhyay, J.M. Petrash, and U.B. Kompella, Nanoparticles in porous microparticles prepared by supercritical infusion and pressure quench technology for sustained delivery of bevacizumab, Mol. Pharm., 10 (2013) 4676-4686.

[15] G. Kogan, L. Soltes, R. Stern, and P. Gemeiner, Hyaluronic acid: a natural biopolymer with a broad range of biomedical and industrial applications, Biotechnol. Lett., 29 (2007) 17-25.

[16] S. Giarra, C. Serri, L. Russo, S. Zeppetelli, R.G. De, A. Borzacchiello, M. Biondi, L. Ambrosio, and L. Mayol, Spontaneous arrangement of a tumor targeting hyaluronic acid shell on irinotecan loaded PLGA nanoparticles, Carbohydr. Polym., 140 (2016) 400-407.

[17] C. Serri, V. Quagliariello, R.V. Iaffaioli, S. Fusco, G. Botti, L. Mayol, and M. Biondi, Combination therapy for the treatment of pancreatic cancer through hyaluronic acid-decorated nanoparticles loaded with quercetin and gemcitabine: A preliminary in vitro study, J. Cell Physiol, 234 (2019) 4959-4969.

[18] D. Maysinger, K. Krieglstein, J. Filipovic-Grcic, M. Sendtner, K. Unsicker, and P. Richardson, Microencapsulated ciliary neurotrophic factor: physical properties and biological activities, Exp. Neurol., 138 (1996) 177-188.

[19] J.M. Itkonen, A. Urtti, L.E. Bird, and S. Sarkhel, Codon optimization and factorial screening for enhanced soluble expression of human ciliary neurotrophic factor in Escherichia coli, BMC. Biotechnol., 14 (2014) 92.

[20] M. Biondi, L. Indolfi, F. Ungaro, F. Quaglia, M.I. La Rotonda, and P.A. Netti, Bioactivated collagenbased scaffolds embedding protein-releasing biodegradable microspheres: tuning of protein release kinetics, J. Mater. Sci. Mater. Med., 20 (2009) 2117-2128.

[21] F. Mollica, M. Biondi, S. Muzzi, F. Ungaro, F. Quaglia, M.I. La Rotonda, and P.A. Netti, Mathematical modelling of the evolution of protein distribution within single PLGA microspheres: prediction of local concentration profiles and release kinetics, J. Mater. Sci. Mater. Med., 19 (2008) 1587-1593.

[22] F. Ungaro, M. Biondi, I. d'Angelo, L. Indolfi, F. Quaglia, P.A. Netti, and M.I. La Rotonda, Microsphereintegrated collagen scaffolds for tissue engineering: effect of microsphere formulation and scaffold properties on protein release kinetics, J. Control Release, 113 (2006) 128-136.

[23] U. Bilati, E. Allemann, and E. Doelker, Poly(D,L-lactide-co-glycolide) protein-loaded nanoparticles prepared by the double emulsion method--processing and formulation issues for enhanced entrapment efficiency, J. Microencapsul., 22 (2005) 205-214. 
[24] M. van de Weert, W.E. Hennink, and W. Jiskoot, Protein instability in poly(lactic-co-glycolic acid) microparticles, Pharm. Res., 17 (2000) 1159-1167.

[25] Q. Xu, N.J. Boylan, J.S. Suk, Y.Y. Wang, E.A. Nance, J.C. Yang, P.J. McDonnell, R.A. Cone, E.J. Duh, and J. Hanes, Nanoparticle diffusion in, and microrheology of, the bovine vitreous ex vivo, J. Control Release, 167 (2013) 76-84.

[26] C. Serri, M. Argiro, L. Piras, D.G. Mita, A. Saija, L. Mita, M. Forte, S. Giarra, M. Biondi, S. Crispi, and L. Mayol, Nano-precipitated curcumin loaded particles: effect of carrier size and drug complexation with (2-hydroxypropyl)-beta-cyclodextrin on their biological performances, Int. J. Pharm., 520 (2017) 21-28.

[27] M.P. Kummer, J.J. Abbott, S. Dinser, and B.J. Nelson, Artificial vitreous humor for in vitro experiments, Conf. Proc. IEEE Eng Med. Biol. Soc., 2007 (2007) 6407-6410.

[28] R.P. Batycky, J. Hanes, R. Langer, and D.A. Edwards, A theoretical model of erosion and macromolecular drug release from biodegrading microspheres, J. Pharm. Sci., 86 (1997) 1464-1477.

[29] P. Blasi, S.S. D'Souza, F. Selmin, and P.P. DeLuca, Plasticizing effect of water on poly(lactide-coglycolide), J. Control Release, 108 (2005) 1-9.

[30] A. Brunner, K. Mader, and A. Gopferich, $\mathrm{pH}$ and osmotic pressure inside biodegradable microspheres during erosion, Pharm. Res., 16 (1999) 847-853.

[31] J. Siepmann and A. Gopferich, Mathematical modeling of bioerodible, polymeric drug delivery systems, Adv. Drug Deliv. Rev., 48 (2001) 229-247.

[32] M.C. Hamoudi-Ben Yelles, T. Tran, V, F. Danede, J.F. Willart, and J. Siepmann, PLGA implants: How Poloxamer/PEO addition slows down or accelerates polymer degradation and drug release, J. Control Release, 253

19-29.

\section{Captions to Figures and Tables}

Figure 1. Examinations with EDS of as-received raw materials, PP MPs and PPHA MPs.

Figure 2. Representative DSC thermograms of as-received raw materials, physical mixtures of poloxamers and PPHA MPs; A: first heating cycles; B: second heating cycles.

Figure 3. SEM images of degrading PPHA MPs at incubation times. 

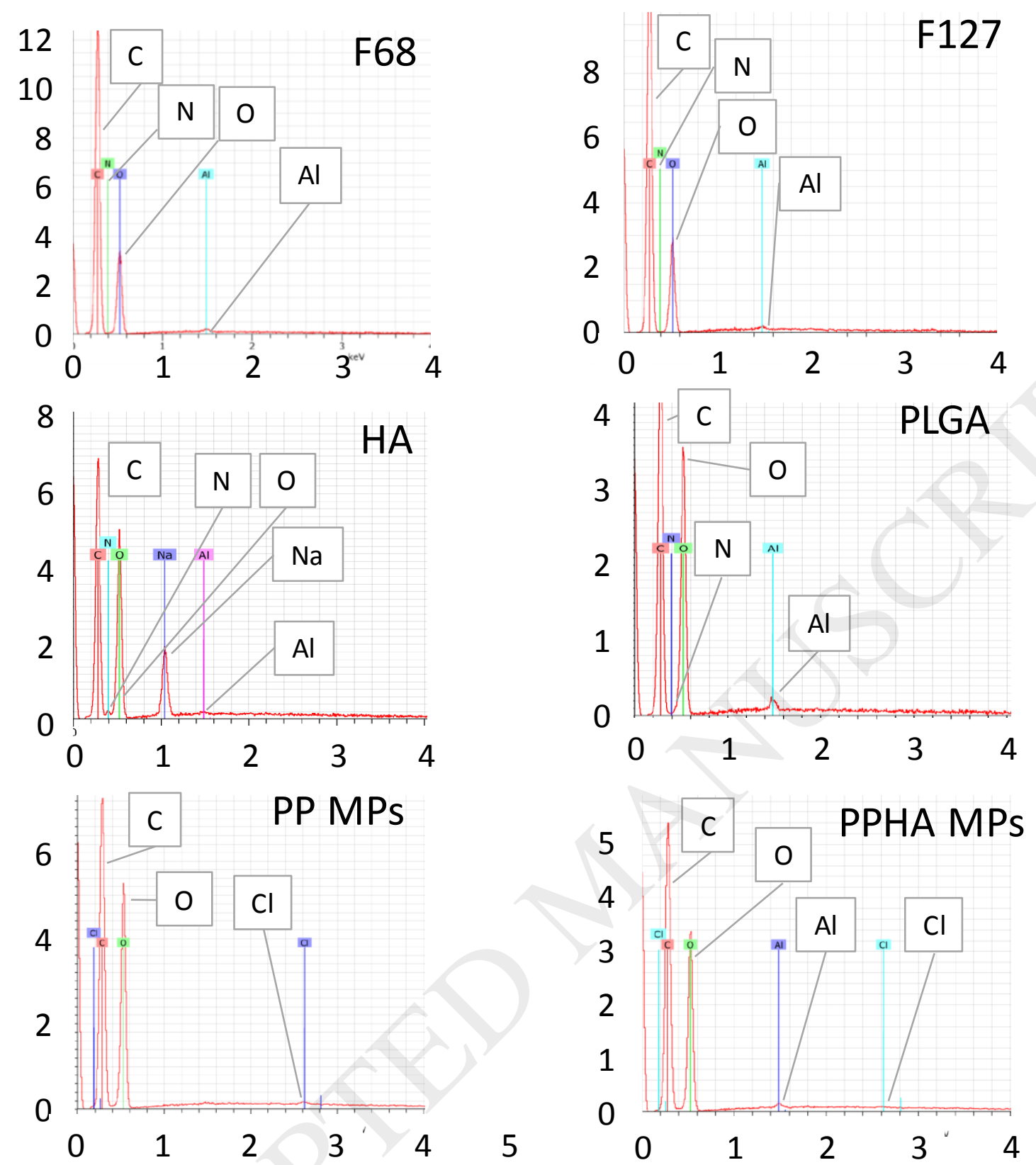

Figure 1. 

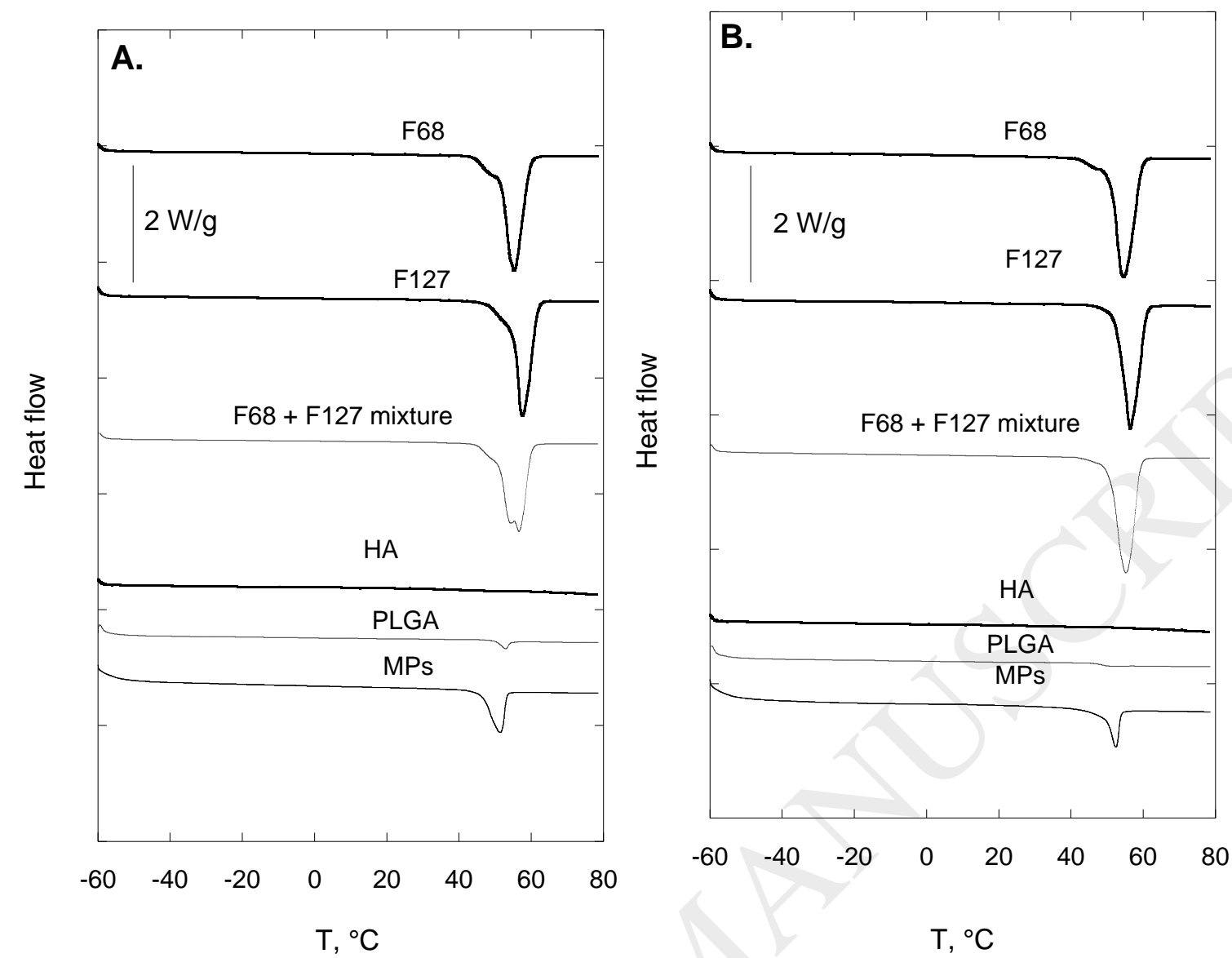

Figure 2. 


\section{HA734}
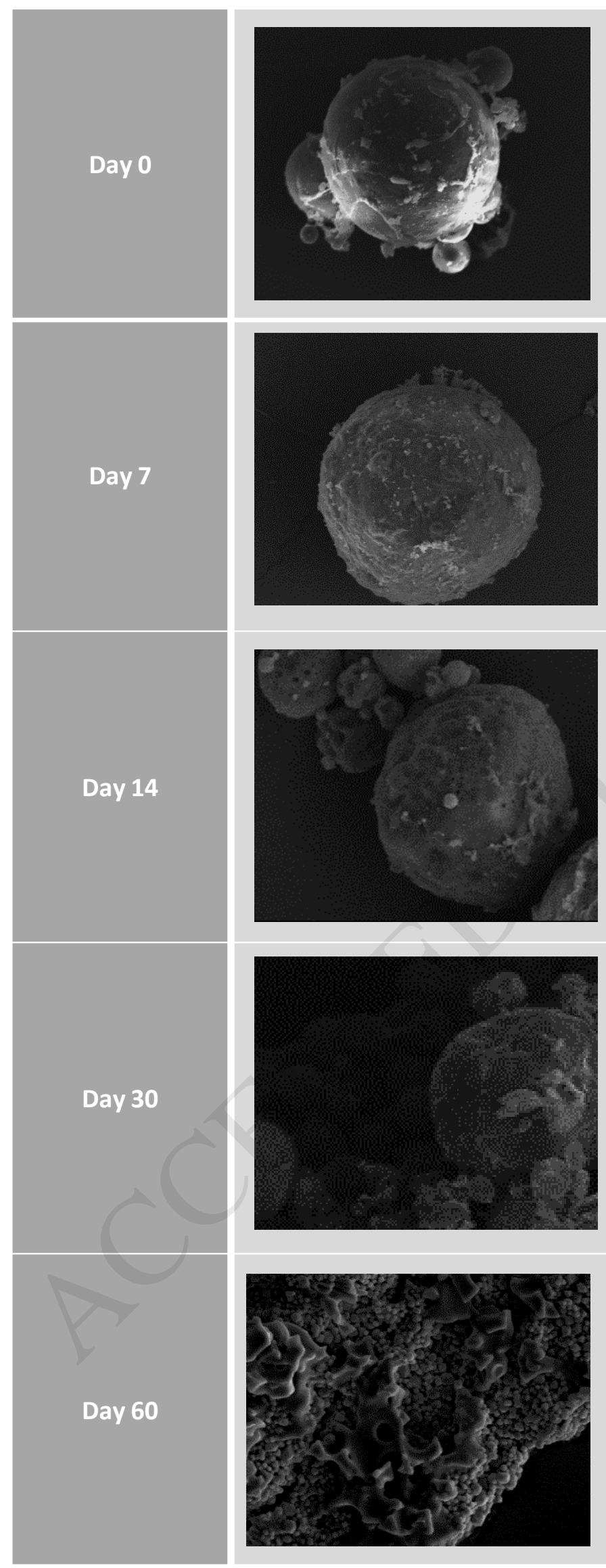


\section{Figure 3.}

Table 1. Elementary composition of raw materials (HA, poloxamers F68 and F127 and PLGA) and of the produced MPs, reported in wt $\%$. Standard deviations were calculated on at least two repeats.

\begin{tabular}{|l|l|l|l|l|l|l|}
\hline & $\mathbf{C}$ & $\mathbf{N}$ & $\mathbf{O}$ & $\mathbf{N a}$ & $\mathbf{A l}$ & $\mathbf{C l}$ \\
\hline PLGA & $47.6 \pm 1.0$ & $1.8 \pm 0.4$ & $50.0 \pm 1.1$ & & $0.6 \pm 0.5$ & \\
\hline F68 & $60.4 \pm 0.8$ & $2.5 \pm 0.4$ & $36.7 \pm 1.0$ & & $0.4 \pm 0.5$ & \\
\hline F127 & $62.2 \pm 0.9$ & $2.1 \pm 0.5$ & $35.5 \pm 0.8$ & & $0.3 \pm 0.3$ & \\
\hline HA734 & $44.6 \pm 1.5$ & $5.2 \pm 0.4$ & $45.0 \pm 1.2$ & $5.2 \pm 0.1$ & $0.1 \pm 0.1$ & \\
\hline PP MPs & $49.0 \pm 0.5$ & & $50.9 \pm 0.5$ & & traces & traces \\
\hline PPHA MPs & $50.8 \pm 0.1$ & & $49.0 \pm 0.1$ & & traces & traces \\
\hline
\end{tabular}

Table 2. Results of thermal analyses on raw materials and MPs. Mean values and standard deviations were calculated from at last three independent replicas.

\begin{tabular}{|l|l|l|l|l|l|l|}
\hline & \multicolumn{5}{l}{ First scan } & \multicolumn{2}{l|}{ Second scan } \\
\hline & $\mathrm{T}_{\mathrm{m}},{ }^{\circ} \mathrm{C}$ & $\mathrm{T}_{\mathrm{g}},{ }^{\circ} \mathrm{C}$ & $\Delta \mathrm{H}_{\mathrm{m}}, \mathrm{J} / \mathrm{g}$ & $\mathrm{T}_{\mathrm{m}},{ }^{\circ} \mathrm{C}$ & $\mathrm{T}_{\mathrm{g}},{ }^{\circ} \mathrm{C}$ & $\Delta \mathrm{H}_{\mathrm{m}}, \mathrm{J} / \mathrm{g}$ \\
\hline F68 & $55.5 \pm 0.5$ & - & $131 \pm 14$ & $54.9 \pm 0.6$ & - & $121 \pm 14$ \\
\hline F127 & $57.1 \pm 0.6$ & - & $128 \pm 2$ & $55.9 \pm 0.7$ & - & $120 \pm 7$ \\
\hline F68 + F127 mixture & $55.7 \pm 0.4^{*}$ & - & $131 \pm 6$ & $55.2 \pm 0.2$ & - & $121 \pm 5$ \\
& $56.8 \pm 0.6 *$ & & & & & \\
\hline HA & - & - & - & - & - & - \\
\hline PLGA & - & $52.1 \pm 0.2$ & - & - & $48.7 \pm 0.3$ & - \\
\hline MPs & $48.2 \pm 3.7$ & N.D. & $17.2 \pm 1.5$ & $51.7 \pm 1.1$ & N.D. & 5.830 .24 \\
\hline
\end{tabular}

${ }^{*}$ First peak

*** Second peak 